\title{
Impact of pre-diagnostic triglycerides and HDL-cholesterol on breast cancer recurrence and survival by breast cancer subtypes
}

Trygve Lofterød ${ }^{1 *}$, Elin S. Mortensen ${ }^{2}$, Hawa Nalwoga ${ }^{3}$, Tom Wilsgaard ${ }^{4}$, Hanne Frydenberg $^{1}$, Terje Risberg ${ }^{5}$, Anne Elise Eggen ${ }^{4}$, Anne McTiernan ${ }^{6}$, Sura Aziz ${ }^{3}$, Erik A. Wist ${ }^{1}$, Andreas Stensvold ${ }^{7}$, Jon B. Reitan ${ }^{1}$, Lars A. Akslen ${ }^{3,8}$ and Inger Thune 1,9

\begin{abstract}
Background: High triglycerides and low levels of high density lipoprotein (HDL)-cholesterol are observed to promote tumor growth. However, whether breast cancer heterogeneity may explain the contradictory influence of triglycerides and cholesterol observed on breast cancer prognosis remains unclear.

Methods: A population-based survival study among 464 breast cancer cases identified within the Troms $\varnothing$ study was conducted. Pre-diagnostic triglycerides, total-cholesterol and HDL-cholesterol were measured, and detailed clinical and histopathological data were obtained. Using tissue microarray, all breast cancer cases were reclassified into the following subtypes: Luminal A, Luminal B, HER2-enriched, and triple negative breast cancer (TNBC). Multivariable Cox proportional hazards regression models were used to study the associations between pre-diagnostic lipids and breast cancer recurrence, mortality, and survival.
\end{abstract}

Results: A total of 464 breast cancer patients, with mean age at diagnosis of 57.9 years, were followed for a mean 8.4 years. TNBC patients in the highest tertile of triglycerides $(\geq 1.23 \mathrm{mmol} / \mathrm{l})$ had 3 times higher overall mortality compared to TNBC patients in the lowest tertile ( $\leq 0.82 \mathrm{mmol} / \mathrm{l})(\mathrm{HR} 2.99,95 \% \mathrm{Cl} 1.17-7.63)$, and the 5-year overall survival was 19\% lower for TNBC patients in the highest vs. lowest tertile of triglycerides (65\% vs. 84\%). TNBC patients in the highest tertile of the $\mathrm{HDL}$-cholesterol/total-cholesterol ratio $(\geq 0.35)$, compared to those in the lowest tertile $(\leq 0.27)$, had a $67 \%$ reduced overall mortality risk (HR 0.33, 95\% Cl 0.12-0.89). No associations were observed between lipids and prognostic outcome among breast cancer patients overall, or among patients with luminal A and luminal B subtypes. Among HER2-enriched patients, pre-diagnostic triglyceride level was inversely associated with overall mortality.

Conclusion: Our study suggests that pre-diagnostic triglycerides and the HDL-cholesterol/total-cholesterol ratio may independently provide unique information regarding prognostic outcome among triple negative breast cancer patients. However, a small sample size underlines the need for additional studies.

Keywords: Breast cancer, Lipids, Molecular subtype, Triple negative breast cancer, Survival

\footnotetext{
* Correspondence: tlofteroed@hotmail.com

'Department of Oncology, Oslo University Hospital, Ullevål, N-0424 Oslo,

Norway

Full list of author information is available at the end of the article
}

(c) The Author(s). 2018 Open Access This article is distributed under the terms of the Creative Commons Attribution 4.0 International License (http://creativecommons.org/licenses/by/4.0/), which permits unrestricted use, distribution, and reproduction in any medium, provided you give appropriate credit to the original author(s) and the source, provide a link to the Creative Commons license, and indicate if changes were made. The Creative Commons Public Domain Dedication waiver (http://creativecommons.org/publicdomain/zero/1.0/) applies to the data made available in this article, unless otherwise stated. 


\section{Background}

Studies investigating the influence of metabolic differences on breast cancer prognosis often show contradictory results, and information on breast cancer heterogeneity/subtypes is not included [1-4]. The identification of distinct breast cancer molecular subtypes has warranted more tailored treatment regimes, and a prolonged survival has been observed for the majority of breast cancer patients, but not for all [5]. Importantly, triple negative breast cancer (TNBC), defined by lack of estrogen receptor (ER), progesterone receptor (PR), and human epidermal growth factor receptor-2 (HER2) expression [6], remains associated with shorter disease-free interval and higher mortality rate [7]. Furthermore, prognostic diversity often exists within each molecular subtype [8], and metabolic heterogeneity is likely to be present in all cancers, including breast cancer [9]. Identifying biologic markers associated with metabolic heterogeneity, breast cancer subtype and prognosis is of importance in order to discover potential targets for treatment and optimize breast cancer outcomes.

Dyslipidemia has been independently linked with breast cancer development [10], but studies are conflicting [11]. Moreover, dyslipidemia is strongly associated with obesity. Recently, patients` body mass index (BMI) at diagnosis was observed to be differently distributed across breast cancer molecular subtypes, and obese women were more likely to have TNBC [12], also supported by others [13, 14]. Of note, obesity has been linked to increased risk of recurrence and breast cancer specific mortality $[15,16]$. However, a survival study newly demonstrated an association between pre-diagnostic obesity and worse outcome only among patients with Luminal A disease [17]. Moreover, previous studies have shown inconsistent results on pre-diagnostic obesity and TNBC prognosis $[18,19]$, suggesting there may be other metabolic mechanisms driving the carcinogenesis in more aggressive tumors. Triglycerides serve as an independent source for fatty acid oxidation [20], an important process promoting cell proliferation and tumor growth [21], proposing a carcinogenic potential of triglycerides. However, the relationship between pre-diagnostic triglycerides and breast cancer development by molecular subtype remains unclear [22]. Moreover, cholesterol has been suggested to play a role in breast cancer progression [23]. Conversely, high density lipoprotein (HDL)-cholesterol possesses anti-inflammatory properties [24], and has been inversely associated with breast cancer risk [25], and suggestively breast cancer survival [22]. We have previously observed that low HDL-cholesterol may be associated with higher estrogen levels and absolute mammographic density [26-28]. In addition, different lipoprotein subfractions vary by progesterone receptor expression [29], suggesting the influence of HDL-cholesterol on breast cancer prognosis may differ by breast cancer phenotype [30].
Thus, the main aim of the present study was to investigate whether variations in pre-diagnostic lipid levels in a population-based breast cancer cohort independently affect breast cancer recurrence and mortality by molecular subtypes.

\section{Material and method \\ Study design, settings and participants}

The present study includes 464 women diagnosed with primary invasive histological verified breast cancer in the period 1980-2014, who participated in the Troms $\varnothing$ Study during 1979-2008 (Tromsø surveys 2-6) [31]. The Tromsø Study is a population-based prospective study aiming to explore risk factors for chronic diseases. Age-eligible women, including total birth cohorts and random samples of the Tromsø population were recruited: A total of 19,947 women participated (77.0\% of invited women).

\section{Assessment of weight, height and serum lipids}

Height and weight were measured upon enrollment in the Troms $\varnothing$ Study (study entry=pre-diagnostic), and BMI $\left(\mathrm{kg} / \mathrm{m}^{2}\right)$ was calculated. All attendees had non-fasting blood samples drawn at each study entry. The samples were analyzed at the Department of Laboratory Medicine, University Hospital North Norway, Tromsø (ISO-standard accredited laboratory). Serum triglycerides, total-cholesterol, and HDL-cholesterol were analyzed within $10 \mathrm{~h}$ by enzymatic, colorimetric methods and commercially available kits (CHOD-PAP for cholesterol, and GPO-PAP for triglycerides; Boehringer Mannheim). Coefficient of variation (CV) 3.3\%. [32]. However, in the Tromsø survey 4 (1994-95), HDL-cholesterol was measured after the precipitation of LDL with heparin and manganese chloride (CV 4.2\%) [31, 32].

\section{Case identification and breast tumor characteristics}

Participants were linked to the Cancer Registry of Norway using the unique national 11-digit identification number (Statistics Norway), and 656 women in the cohort were identified and diagnosed with primary invasive breast cancer from 1979 through December 31st 2014. To account for the possibility that undiagnosed cancer could influence our results, we excluded those in whom breast cancer developed during the first year after they entered the cohort $\left(\mathrm{n}_{\text {cases }}=12\right)$. We also excluded those being $<20$ years of age at study entry $\left(\mathrm{n}_{\text {cases }}=3\right)$, and those with missing information on tumor cell proliferation marker, Ki67 hotspot index $\left(\mathrm{n}_{\text {cases }}=123\right)$, ER, PR, or HER2 status $\left(\mathrm{n}_{\text {cases }}=54\right)$. Hence, a total of 464 women with histologically verified invasive primary breast cancer were included. 
All tumor samples were fixed in $4 \%$ buffered formaldehyde before processing and embedding in paraffin. In order to obtain comparable results due to changes in identifying tumor characteristics over time (1980-2012), and to obtain complete information on tumor characteristics, a majority $\left(\mathrm{n}_{\text {cases }}=375\right)$ of the tissue samples were analyzed on tissue microarrays (TMA) (Centre for Cancer Biomarkers CCBIO, Section for Pathology, University of Bergen). Immunohistochemistry (IHC) and HER2 Silver in Situ Hybridization (SISH) were employed in TMA, to obtain Ki67 and HER2 status, respectively. HER2-SISH was performed on IHC 2+ cases, and considered positive if HER2/Chr17 ratio by SISH was $>2.0$. Tumors were stained for ER and PR, and considered positive if $\geq 10 \%$ of tumor nuclei stained positive [33]. Breast tumors diagnosed after $2012\left(\mathrm{n}_{\text {cases }}=89\right)$ were analyzed using immunohistochemistry for hormone receptor status and Ki67, and immunohistochemistry and fluorescence in situ hybridization for HER2.

We classified the breast tumors into molecular subtypes (13th St Gallen International Breast Cancer Expert Panel) [34] as follows: Luminal A - ER positive, PR positive, HER2 negative, and Ki-67<20\%; Luminal B - ER positive and/or PR positive, HER2 positive (or HER2 negative and Ki-67 $\geq 20 \%$ or PR negative); HER2-enriched - ER negative, PR negative, and HER2 positive; and TNBC - ER negative, PR negative and HER2 negative.

\section{Outcomes: Mortality and recurrence}

Person-time of follow-up was calculated from the date of breast cancer diagnosis until date of recurrence, death, time of emigration, or end of follow-up (December 31st, 2014), whichever event occurred first. We obtained information on death and emigration through linkage to the Norwegian Cause of Death Registry and Statistics Norway. The mean interval between the pre-diagnostic measurements and diagnosis was 18.6 years (standard deviation (SD) 9.23, range 1.0135.2 years).

We used the following outcomes: 1) overall mortality and overall survival: death of any cause, and the time interval from date of diagnosis to death of any cause, respectively (all breast cancer stages included, stages 1-4); 2) breast cancer-free interval: the time interval from date of diagnosis to breast cancer recurrence or breast cancer mortality (stages $1-3$ breast cancer at diagnosis).

\section{Treatment data}

Each patient's medical chart was reviewed. Details of all treatment regimens were obtained, including type of surgery, chemotherapy, radiotherapy, and endocrine treatment. Date and site of recurrence was collected, and recurrence was defined by local, regional, and/or visceral relapse.

\section{Statistical methods}

We used multivariable Cox proportional hazards regression models to study the association of pre-diagnostic lipid levels on overall mortality and breast cancer-free interval by molecular subtypes. In order to study the association between lipids and outcomes (overall mortality and breast cancer-free interval) among patients with HER2 positive $(+)$ breast cancer, we combined the patients with HER2+ expression from Luminal B subtype and the patients from HER2-enriched subtype.

We categorized lipids into the following groups by tertiles: triglycerides $(\leq 0.82,0.83-1.22$, and $\geq 1.23 \mathrm{mmol} / \mathrm{l})$, total-cholesterol $(\leq 5.14,5.15-6.25$, and $\geq 6.26 \mathrm{mmol} / \mathrm{l})$, and HDL-cholesterol/total-cholesterol ratio $(\leq 0.27$, $0.28-0.34$, and $\geq 0.35$ ). The triglyceride/HDL-cholesterol ratio (tertile splits: $\leq 0.47,0.48-0.75$, and $\geq 0.76$ ) was used as a surrogate marker for insulin resistance [35].

Based on our previous results [36], and in order to evaluate our present findings, a total of 57 breast cancer cases were checked for agreement between molecular subtyping based on immunohistochemistry and TMA. We observed an agreement between these two methods in $73 \%$ of the breast cancer cases (kappa value 0.63 ).

Based on suggested biological mechanisms influencing serum lipid levels, breast cancer recurrence and overall and breast cancer specific mortality, several variables were included in the Cox proportional hazard model as potential confounders. Age (continuous), BMI (continuous), and smoking habits (categorical) at blood sampling, age at diagnosis (continuous) and disease stage (categorical) were included as covariates in the final models. To account for secular trends in treatment [http://www.nbcg.no], we adjusted for year of diagnosis, chemotherapy (yes/no) and endocrine treatment (yes/no), but our observations were not significantly influenced. In addition, in order to account for comorbid disease confounding, we adjusted for alcohol habits, physical activity, blood glucose, blood pressure, and time since last meal. Moreover, we excluded women who died within the first and third year after being diagnosed breast cancer to account for serious comorbidity at diagnosis. However, none of these adjustments influenced our results, and they were not included in the final models. In our study including 464 breast cancer patients with 129 events we have $80 \%$ power to detect a hazard ratio of 1.28 per standard deviation increase in a continuous variable, and $80 \%$ power to detect a hazard ratio of 1.63 for an equally distributed binary variable. Consequently, including 40 events, the respective hazard ratios are 1.56 and 2.43 .

To test for interaction between lipids and breast cancer molecular subtypes, two-way cross product terms were 
added to logistic regression and cox regression models. In both logistic and cox regression models, we observed statistically significant interaction between triglycerides and TNBC ( $p=0.042$ and $p=0.025$, respectively), and between the HDL-cholesterol/total-cholesterol ratio and TNBC $(p=0.033$ and $p=0.065$, respectively) (data not shown in table). The proportional hazards assumption was assessed by visual inspection of log-log survival functions of levels of pre-diagnostic lipids. Among TNBC, log rank-tests were performed to compare differences in survival curves between the tertiles of triglycerides $(p<0.001)$. All statistical tests were two-sided using a significance level of $p<$ 0.05, and analysis was conducted using SPSS version 21.0.

In order to increase the sample size to $n_{\text {cases }}=641$, we used multiple imputation and imputed values in 20 datasets on the following variables: Ki-67, ER-, PR-, and HER2 status, age, disease stage, current smoking, BMI, triglycerides, $\mathrm{HDL} /$ total-cholesterol ratio, and total-cholesterol. Separate Cox regression analyses showed results that were not substantially different from what we observed in our complete case analyses, and these data are not presented in the text (TNBC patients presented in Additional file 1: Table S1).

\section{Results}

\section{Characteristics of the study population}

Of the 464 women diagnosed with invasive histologically verified breast cancer (mean age at diagnosis of 57.9 years), a total of 129 died during a mean follow-up of 8.4 years: $51.9 \%$ were attributable to breast cancer, $7.9 \%$ to other cancers, $7.1 \%$ to cardiovascular disease, $16.4 \%$ to other causes, and $16.7 \%$ to unregistered cause of death. Molecular subtypes were distributed as follows: Luminal A (49.1\%), Luminal B (21.3\%), HER2-enriched (9.3\%) and TNBC (20.3\%). Patients diagnosed with the most common subtype, Luminal A, in comparison to patients diagnosed with TNBC, were older at diagnosis (58.8 years vs. 55.9 years, $p=0.035$ ), had smaller tumors (21.6 mm vs. $29.2 \mathrm{~mm}, p=0.001)$, lower Ki-67 (8.43\% vs. $30.5 \%, p<0.001)$, and lower overall mortality $(19.7 \%$ vs. $40.4 \%, p<0.001$ ) (Table 1). Characteristics of the 123 women excluded due to missing information on Ki-67 did not substantially differ from the final study population (data not shown).

\section{5-year overall survival and 5-year breast cancer-free interval by molecular subtypes}

The 5-year overall survival for the entire cohort was $83 \%$ (data not shown in tables or figures). When stratified by tumor subtypes, women with Luminal A breast cancer had a 5 -year overall survival of $89 \%$, and a 5 -year breast cancer-free interval of 92\%. Those with HER2-enriched and TNBC subtypes had 5-year overall survival of 73 and $75 \%$, respectively, and 5 -year breast cancer-free interval of 74 and $84 \%$, respectively (Additional file 2: Figure S1 and Additional file 3: Figure S2).

\section{Pre-diagnostic lipids and total mortality}

Table 2 presents the multivariable adjusted hazard ratios (HRs) for all-cause mortality in relation to pre-diagnostic lipids and breast cancer molecular subtypes. No association was observed between triglycerides, total-cholesterol, the HDL-cholesterol/total-cholesterol ratio, and the triglyceride/HDL-cholesterol ratio and overall mortality among all breast cancer patients combined, or among Luminal A and B subtypes.

Compared to patients with TNBC in the lowest tertile of triglycerides, those in the highest tertile $(\geq 1.23 \mathrm{mmol} / \mathrm{l})$ had a 3 times higher overall mortality (HR 2.99, 95\% CI 1.17-7.63) (Table 2) and 19\% lower 5-year overall survival (65\% vs. 84\%) (Fig. 1). TNBC patients in the highest $(\geq 0.35)$ vs. lowest $(0.27)$ tertile of the HDL/total-cholesterol ratio, had a $67 \%$ reduced overall mortality risk (HR 0.33, 95\% CI 0.12-0.89) (Table 2).

Among women with HER2+ breast cancer, those in the highest vs. lowest tertile of triglycerides had an $86 \%$ reduced overall mortality risk (HR 0.14 , 95\% CI $0.03-$ 0.60, p-trend 0.038) (Additional file 4: Table S2).

The triglyceride/HDL-cholesterol ratio was positively associated with overall mortality among TNBC patients (Additional file 5: Table S3).

\section{Pre-diagnostic lipids and breast cancer-free interval}

Table 3 presents the multivariable HRs for breast cancer-free interval by pre-diagnostic lipids and molecular subtypes. We observed no association between lipid levels and breast cancer-free interval among all breast cancer patients combined, or among Luminal A, Luminal B, or HER2-enriched molecular subtypes.

Among TNBC, women in the highest $(\geq 1.23 \mathrm{mmol} / \mathrm{l})$ vs. lowest $(\leq 0.82 \mathrm{mmol} / \mathrm{l})$ tertile of triglycerides had 5.6 times higher risk for recurrence or death from breast cancer (HR 5.63, 95\% CI 1.64-19.3) (Table 3), and the 5 -year breast cancer-free interval was $24 \%$ lower for women in the highest vs. lowest tertile of triglycerides (69\% vs. 93\%) (Fig. 2).

No associations were observed between triglycerides and breast cancer-free interval among HER2+ patients (Additional file 5: Table S3), or between total-cholesterol and breast cancer-free interval overall or by molecular subtypes (data not presented in table).

\section{Discussion}

We observed strong associations between pre-diagnostic triglycerides and overall mortality and breast cancer-free interval among TNBC patients, and the 5-year overall survival was $19 \%$ lower for patients in the highest tertile of triglycerides compared to those in the lowest tertile of 
Table 1 Descriptive characteristics among breast cancer patients by molecular subtypes

\begin{tabular}{|c|c|c|c|c|c|}
\hline \multirow[t]{2}{*}{ Molecular subtypes } & All cases $(N=464)^{a}$ & Luminal A $(N=228)^{a}$ & Luminal B $(N=99)^{a}$ & HER-2 enriched $(N=43)^{a}$ & $\operatorname{TNBC}(N=94)^{\mathrm{a}}$ \\
\hline & Mean (SD) / \% & Mean (SD) / \% & Mean (SD) / \% & Mean (SD) / \% & Mean (SD) / \% \\
\hline \multicolumn{6}{|l|}{ Characteristics at study entry } \\
\hline Age at blood sampling, years & $39.5(14.5)$ & $40.3(15.2)$ & $36.5(10.7)$ & $41.2(15.1)$ & $39.9(14.7)$ \\
\hline Age at diagnosis, years & $57.9(12.6)$ & $58.8(12.8)$ & $57.0(10.3)$ & $59.0(13.3)$ & $55.9(13.8)$ \\
\hline Follow-up after diagnosis, years & $8.43(6.93)$ & $9.35(6.78)$ & $6.43(5.59)$ & $7.27(6.83)$ & $8.72(8.23)$ \\
\hline \multicolumn{6}{|l|}{ Clinical variables $^{\mathrm{b}}$} \\
\hline $\mathrm{SBP}, \mathrm{mmHg}$ & $125(19.4)$ & $125(19.5)$ & $123(15.9)$ & $129(24.5)$ & $126(20.3)$ \\
\hline Waist-hip ratio & $0.83(0.07)$ & $0.84(0.08)$ & $0.81(0.06)$ & $0.82(0.05)$ & $0.84(0.07)$ \\
\hline $\mathrm{BMI}, \mathrm{kg} / \mathrm{m}^{2}$ & $23.4(3.68)$ & $23.3(3.26)$ & $23.3(3.50)$ & $23.3(3.86)$ & $24.1(4.80)$ \\
\hline \multicolumn{6}{|l|}{ Reproductive factors $^{\mathrm{b}}$} \\
\hline Number of children, number & $1.93(1.39)$ & $1.88(1.33)$ & $1.63(1.11)$ & $2.40(1.96)$ & $2.26(1.53)$ \\
\hline Age at menarche, years & $13.3(1.39)$ & $13.2(1.50)$ & $13.4(1.28)$ & $13.2(1.09)$ & $13.4(1.30)$ \\
\hline HRT use, $\%$ & 30.5 & 31.5 & 28.7 & 28.6 & 30.5 \\
\hline \multicolumn{6}{|l|}{ Serum samples ${ }^{\mathrm{b}}$} \\
\hline Total cholesterol, mmol/l & $5.82(1.30)$ & $5.76(1.22)$ & $5.77(1.30)$ & $6.03(1.32)$ & $5.95(1.46)$ \\
\hline HDL-cholesterol, mmol// & $1.75(0.40)$ & $1.76(0.40)$ & $1.72(0.32)$ & $1.67(0.38)$ & $1.78(0.45)$ \\
\hline Triglycerides, mmol// & $1.16(0.76)$ & $1.11(0.59)$ & $1.12(0.53)$ & $1.35(1.23)$ & $1.28(1.14)$ \\
\hline \multicolumn{6}{|l|}{ Lifestyle factors $^{\mathrm{b}}$} \\
\hline Moderate physical activity, \% & 72.5 & 72.8 & 75.3 & 72.7 & 68.2 \\
\hline Current smokers, \% & 40.4 & 40.4 & 38.1 & 48.5 & 40.0 \\
\hline \multicolumn{6}{|l|}{ Tumor characteristics } \\
\hline Tumor size, mm & $24.6(19.5)$ & $21.6(18.6)$ & $26.1(17.8)$ & $30.3(19.1)$ & $29.2(23.0)$ \\
\hline Number of metastatic lymph nodes & $2.03(3.92)$ & $1.46(3.23)$ & $3.04(4.66)$ & $2.00(2.97)$ & $2.43(4.67)$ \\
\hline Stage, $\%$ & $1.77(0.81)$ & $1.59(0.68)$ & $1.92(0.80)$ & $2.12(0.96)$ & $1.95(0.93)$ \\
\hline 1 & 41.7 & 51.3 & 31.6 & 27.9 & 35.5 \\
\hline 2 & 39.6 & 38.9 & 49.0 & 30.2 & 35.5 \\
\hline 3 & 15.7 & 8.80 & 15.3 & 37.2 & 22.9 \\
\hline 4 & 3.00 & 0.90 & 4.10 & 4.70 & 6.50 \\
\hline Histologic grading, \% & $1.92(0.73)$ & $1.55(0.57)$ & $2.13(0.68)$ & $2.54(0.51)$ & $2.49(0.65)$ \\
\hline 1 & 30.1 & 48.6 & 17.8 & 2.60 & 8.60 \\
\hline 2 & 45.8 & 47.6 & 53.3 & 42.1 & 34.6 \\
\hline 3 & 24.1 & 3.80 & 28.9 & 55.3 & 56.8 \\
\hline Estrogen positive, \% & 64.2 & 91.2 & 88.7 & 0 & 0 \\
\hline Progesterone positive, $\%$ & 47.9 & 71.6 & 61.3 & 0 & 0 \\
\hline HER2 positive, $\%$ & 16.0 & 0 & 34.0 & 100 & 0 \\
\hline $\mathrm{Ki}-67 \%$ & $18.9(17.3)$ & $8.43(4.98)$ & $30.4(16.1)$ & $27.9(17.1)$ & $30.5(22.0)$ \\
\hline \multicolumn{6}{|l|}{ Treatment } \\
\hline \multicolumn{6}{|l|}{ Type of surgery } \\
\hline Breast conserving surgery & 45.6 & 54.5 & 45.1 & 33.0 & 27.0 \\
\hline Mastectomy & 52.5 & 44.6 & 51.6 & 66.7 & 70.3 \\
\hline Others $^{c}$ & 1.90 & 0.90 & 3.30 & 0.30 & 2.70 \\
\hline Chemotherapy, \% & 38.2 & 25.9 & 52.6 & 51.5 & 49.4 \\
\hline Radiation therapy, \% & 48.6 & 66.8 & 67.0 & 39.4 & 54.1 \\
\hline Endocrine therapy, \% & 50.3 & 55.7 & 70.1 & 21.2 & 24.7 \\
\hline
\end{tabular}


Table 1 Descriptive characteristics among breast cancer patients by molecular subtypes (Continued)

\begin{tabular}{|c|c|c|c|c|c|}
\hline \multirow[t]{2}{*}{ Molecular subtypes } & All cases $(N=464)^{a}$ & Luminal A $(N=228)^{a}$ & Luminal B $(N=99)^{\text {a }}$ & HER-2 enriched $(N=43)^{\mathrm{a}}$ & $\operatorname{TNBC}(N=94)^{\mathrm{a}}$ \\
\hline & Mean (SD) / \% & Mean (SD) / \% & Mean (SD) / \% & Mean (SD) / \% & Mean (SD) / \% \\
\hline \multicolumn{6}{|l|}{ Outcome } \\
\hline Recurrence, \% & 17.0 & 13.2 & 17.2 & 30.2 & 21.0 \\
\hline Overall mortality, \% & 27.8 & 19.7 & 29.3 & 39.5 & 40.4 \\
\hline Breast cancer mortality, \% & 14.4 & 5.27 & 20.2 & 27.9 & 24.5 \\
\hline
\end{tabular}

${ }^{a}$ Numbers may vary due to missing information

${ }^{\mathrm{b}}$ Clinical variables, reproductive factors, serum samples, and lifestyle factors at study entry

'Other types of surgery include primary reconstruction and oncoplastic surgery

Abbreviations: $B M I$ body mass index, $H D L$ high density lipoprotein, $H R T$ hormone replacement therapy, IDC invasive ductal carcinoma, ILC invasive lobular carcinoma, $n$ number of cases, SPB systolic blood pressure

triglycerides (65\% vs. $84 \%)$. Women with TNBC and in the highest tertile of triglycerides had $24 \%$ lower 5 -year breast cancer-free interval (69\% vs. 93\%) compared to those in the lowest tertile. Conversely, among patients with HER2+ disease, a high serum level of triglycerides was inversely associated with overall mortality. Additionally, the HDL-cholesterol/total-cholesterol ratio was inversely associated with overall mortality among TNBC patients.
Observations in the present study extend previous observations linking variation in pre-diagnostic serum lipids, and in particular triglycerides, to breast cancer prognosis by molecular subtypes. Recently, a Chinese retrospective case series including 221 lean $(\mathrm{BMI}<$ $25 \mathrm{~kg} / \mathrm{m}^{2}$ ) TNBC patients observed that high triglyceride/HDL-cholesterol ratio, but not triglyceride, was associated with poor overall survival [37]. In another case

Table 2 Multivariable adjusted hazard ratios for overall mortality by pre-diagnostic lipids and breast cancer molecular subtypes

\begin{tabular}{|c|c|c|c|c|c|c|c|c|c|c|}
\hline & \multicolumn{2}{|c|}{ All cases (events $\left.{ }^{\mathrm{a}}=129\right)$} & \multicolumn{2}{|c|}{ Luminal $\mathrm{A}$ (events ${ }^{\mathrm{a}}=45$ ) } & \multicolumn{2}{|c|}{ Luminal B (events $\left.{ }^{\mathrm{a}}=29\right)$} & \multicolumn{2}{|c|}{ HER2-enriched (events ${ }^{a}=17$ ) } & \multicolumn{2}{|c|}{ TNBC $\left(\right.$ events $\left.^{\mathrm{a}}=38\right)$} \\
\hline & $n$ & HR $(95 \% \mathrm{Cl})$ & $n$ & HR $(95 \% \mathrm{Cl})$ & $n$ & HR $(95 \% \mathrm{Cl})$ & $n$ & $\mathrm{HR}(95 \% \mathrm{Cl})$ & $n$ & $\mathrm{HR}(95 \% \mathrm{Cl})$ \\
\hline \multicolumn{11}{|l|}{ Triglycerides } \\
\hline Continuous, $\mathrm{mmol} / \mathrm{l}$ & 464 & $1.01(0.88-1.22)$ & 228 & $0.99(0.56-1.75)$ & 99 & $1.64(0.78-3.46)$ & 43 & $0.06(0.01-0.77)$ & 94 & $1.02(0.83-1.24)$ \\
\hline \multicolumn{11}{|l|}{ Tertiles } \\
\hline$\leq 0.82 \mathrm{mmol} / \mathrm{l}$ & 153 & 1.00 & 77 & 1.00 & 33 & 1.00 & 14 & 1.00 & 29 & 1.00 \\
\hline $0.83-1.22 \mathrm{mmol} / \mathrm{l}$ & 158 & $0.69(0.42-1.11)$ & 78 & $1.19(0.53-2.66)$ & 33 & $0.50(0.17-1.46)$ & 14 & $0.13(0.03-0.64)$ & 33 & $0.96(0.35-2.62)$ \\
\hline$\geq 1.23 \mathrm{mmol} / \mathrm{l}$ & 153 & $1.24(0.78-1.99)$ & 73 & $1.26(0.57-2.78)$ & 33 & $1.01(0.36-2.78)$ & 15 & $0.06(0.01-0.55)$ & 32 & $2.99(1.17-7.63)$ \\
\hline p-trend & & 0.351 & & 0.838 & & 0.179 & & 0.001 & & 0.011 \\
\hline \multicolumn{11}{|l|}{ Total-cholesterol } \\
\hline Continuous, mmol// & 464 & $1.03(0.90-1.19)$ & 228 & $1.06(0.83-1.36)$ & 99 & $1.00(0.66-1.51)$ & 43 & $1.05(0.70-1.57)$ & 94 & $1.05(0.77-1.44)$ \\
\hline \multicolumn{11}{|l|}{ Tertiles } \\
\hline$\leq 5.14 \mathrm{mmol} / \mathrm{l}$ & 155 & 1.00 & 77 & 1.00 & 37 & 1.00 & 11 & 1.00 & 30 & 1.00 \\
\hline $5.15-6.25 \mathrm{mmol} / \mathrm{l}$ & 156 & $1.33(0.80-2.22)$ & 82 & $1.66(0.70-3.95)$ & 27 & $0.85(0.29-2.50)$ & 16 & $1.28(0.18-7.93)$ & 31 & $1.01(0.39-2.67)$ \\
\hline$\geq 6.26 \mathrm{mmol} / \mathrm{l}$ & 153 & $1.46(0.87-2.44)$ & 69 & $1.48(0.61-3.60)$ & 35 & $1.20(0.38-3.72)$ & 16 & $2.04(0.34-12.3)$ & 33 & $1.41(0.49-4.06)$ \\
\hline p-trend & & 0.354 & & 0.512 & & 0.830 & & 0.533 & & 0.731 \\
\hline \multicolumn{11}{|c|}{ HDL-cholesterol/total-cholesterol ratio } \\
\hline Continuous, & 464 & $0.89(0.58-1.36)$ & 228 & $0.68(0.02-25.0)$ & 99 & $0.23(0.01-54.2)$ & 43 & $693(0.06-747)$ & 94 & $0.02(0.00-1.39)$ \\
\hline \multicolumn{11}{|l|}{ Tertiles } \\
\hline$\leq 0.27$ & 154 & 1.00 & 76 & 1.00 & 32 & 1.00 & 21 & 1.00 & 25 & 1.00 \\
\hline $0.28-0.34$ & 154 & $0.84(0.65-1.29)$ & 67 & $0.81(0.37-1.79)$ & 37 & $1.48(0.57-3.87)$ & 14 & $1.53(0.45-5.23)$ & 36 & $0.53(0.24-1.19)$ \\
\hline$\geq 0.35$ & 156 & $0.82(0.51-1.33)$ & 85 & $1.05(0.43-2.00)$ & 30 & $0.96(0.33-2.74)$ & 8 & $5.61(0.62-21.0)$ & 33 & $0.33(0.12-0.89)$ \\
\hline $\mathrm{p}$-trend & & 0.385 & & 0.819 & & 0.986 & & 0.176 & & 0.026 \\
\hline
\end{tabular}

Multivariable Cox proportional hazard regression models

${ }^{a}$ Number of deaths

Adjusted for age (continuous), Body mass index (continuous), and current smoking (categorical) at blood sampling, age at diagnosis (continuous), and disease stage (categorical)

Abbreviations: $H D L$ high density lipoprotein, $H R$ hazard ratio; $n$ number of cases, $t$ Total, TNBC triple negative breast cancer 


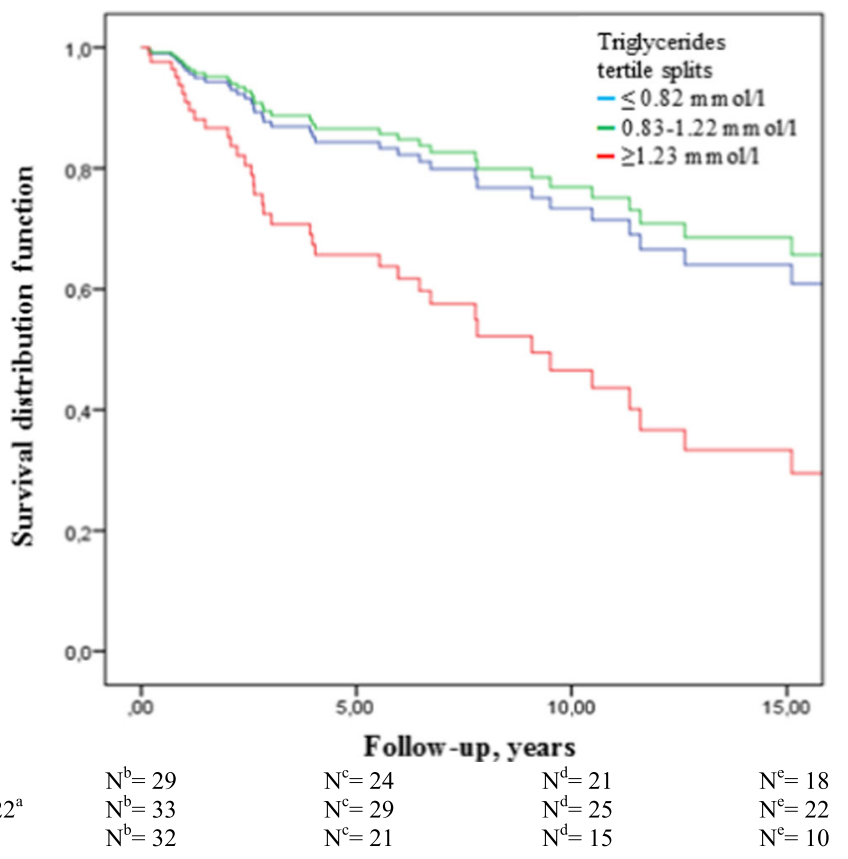

Fig. 1 Overall survival among triple negative breast cancer (TNBC) patients by tertiles of triglycerides. ${ }^{a} \mathrm{mmol} / \mathrm{l},{ }^{\mathrm{b}}$ Number of cases at diagnosis, ${ }^{c}$ Number of cases in follow-up after 5 years, ${ }^{d}$ Number of cases in follow-up after 10 years, ${ }^{e}$ Number of cases in follow-up after 15 years. Abbreviations: $N$, number of cases; TGA, triglycerides

Table 3 Multivariable adjusted hazard ratios for breast cancer-free interval ${ }^{\mathrm{a}}$ by pre-diagnostic lipids and molecular subtypes

\begin{tabular}{|c|c|c|c|c|c|c|c|c|c|c|}
\hline & \multicolumn{2}{|c|}{ All cases (events $\left.{ }^{\mathrm{b}}=91\right)$} & \multicolumn{2}{|c|}{ Luminal A (events ${ }^{\mathrm{b}}=31$ ) } & \multicolumn{2}{|c|}{ Luminal $B\left(\right.$ events $\left.{ }^{\mathrm{b}}=21\right)$} & \multicolumn{2}{|c|}{ HER2-enriched (events ${ }^{\mathrm{b}}=15$ ) } & \multicolumn{2}{|c|}{ TNBC (events $=24)$} \\
\hline & $\mathrm{N}$ & HR $(95 \% \mathrm{Cl})$ & $n$ & HR $(95 \% \mathrm{Cl})$ & $n$ & HR $(95 \% \mathrm{Cl})$ & $n$ & HR $(95 \% \mathrm{Cl})$ & $n$ & HR $(95 \% \mathrm{Cl})$ \\
\hline \multicolumn{11}{|l|}{ Triglycerides } \\
\hline Continuous, mmol/l & 446 & $1.01(0.88-1.22)$ & 224 & $0.88(0.40-1.92)$ & 94 & $0.76(0.23-2.46)$ & 41 & $1.01(0.71-1.47)$ & 87 & $1.12(0.73-1.64)$ \\
\hline \multicolumn{11}{|l|}{ Tertiles } \\
\hline$\leq 0.82 \mathrm{mmol} / \mathrm{l}$ & 148 & 1.00 & 76 & 1.00 & 33 & 1.00 & 13 & 1.00 & 26 & 1.00 \\
\hline $0.83-1.22 \mathrm{mmol} / \mathrm{l}$ & 152 & $0.81(0.47-1.40)$ & 77 & $1.26(0.51-3.11)$ & 31 & $0.24(0.06-0.89)$ & 13 & $0.27(0.04-1.91)$ & 31 & $1.37(0.37-4.98)$ \\
\hline$\geq 1.23 \mathrm{mmol} / \mathrm{l}$ & 146 & $1.23(0.71-2.13)$ & 71 & $1.02(0.38-2.68)$ & 30 & $0.42(0.12-1.52)$ & 15 & $0.26(0.03-2.54)$ & 30 & $5.63(1.64-19.3)$ \\
\hline p-trend & & 0.308 & & 0.854 & & 0.081 & & 0.422 & & 0.004 \\
\hline \multicolumn{11}{|c|}{ HDL-cholesterol/total-cholesterol ratio } \\
\hline Continuous & 446 & $1.18(0.09-15.7)$ & 224 & $16.4\left(0.18-1.4 e^{4}\right)$ & 94 & $15.0\left(0.04-6.2 e^{4}\right)$ & 41 & $116\left(0.00-4.3 e^{6}\right)$ & 87 & $0.03(0.00-6.86)$ \\
\hline \multicolumn{11}{|l|}{ Tertiles } \\
\hline$\leq 0.27$ & 147 & 1.00 & 74 & 1.00 & 29 & 1.00 & 21 & 1.00 & 23 & 1.00 \\
\hline $0.28-0.34$ & 149 & $1.16(0.70-1.93)$ & 66 & $1.52(0.58-4.02)$ & 36 & $5.13(0.98-20.7)$ & 13 & $1.27(0.31-5.21)$ & 34 & $0.58(0.22-1.56)$ \\
\hline$\geq 0.35$ & 150 & $1.02(0.58-1.78)$ & 84 & $1.87(0.69-5.07)$ & 29 & $2.06(0.55-7.75)$ & 7 & $2.99(0.43-20.8)$ & 30 & $0.32(0.10-1.06)$ \\
\hline p-trend & & 0.808 & & 0.219 & & 0.344 & & 0.310 & & 0.060 \\
\hline
\end{tabular}

Multivariable Cox proportional hazard regression models

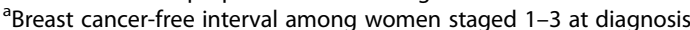

${ }^{\mathrm{b}}$ Number of breast cancer patients with recurrence or death from breast cancer

Adjusted for age (continuous), Body mass index (continuous), and current smoking (categorical) at blood sampling, age at diagnosis (continuous), and disease stage (categorical)

Abbreviations: HDL high density lipoprotein, $H R$ hazard ratio, $n$ number of cases, $T N B C$ triple negative breast cancer 


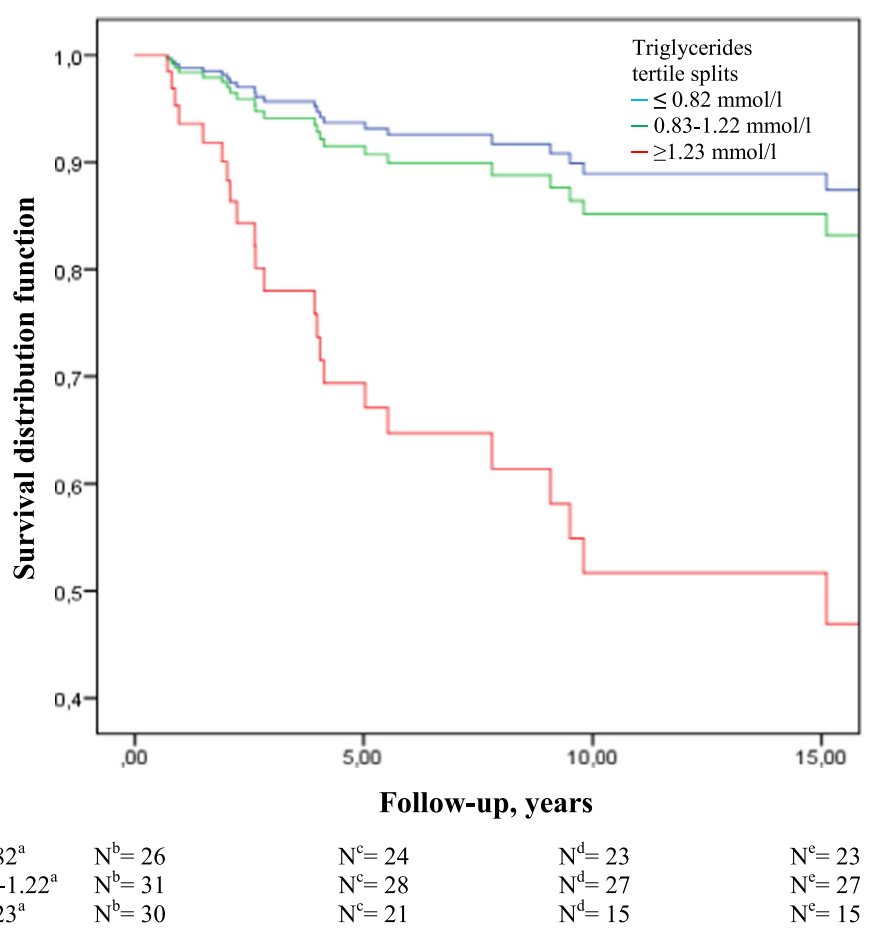

Fig. 2 Breast cancer-free interval among triple negative breast cancer (TNBC) patients by tertiles of triglycerides. ${ }^{a} \mathrm{mmol} / \mathrm{l}$, ${ }^{\mathrm{b}} \mathrm{Number}$ of cases at diagnosis, ${ }^{C}$ Number of breast cancer-free cases in follow-up after 5 years, ${ }^{\mathrm{d}}$ Number of breast cancer-free cases in follow-up after 10 years,

eNumber of breast cancer-free cases in follow-up after 15 years. Abbreviations: N, number of cases; TGA, triglycerides

series, an inverse association was observed between HDL-cholesterol at diagnosis of breast cancer and recurrence and overall mortality among 394 TNBC patients, but there was no association between triglycerides and breast cancer outcomes [30]. Another Chinese study including 1044 breast cancer patients, unclassified by molecular subtype, observed that preoperative lower triglycerides and HDL-cholesterol were associated with shorter disease-free and worse overall survival, respectively [22]. In contrast to our study, triglycerides and HDL-cholesterol were measured at diagnosis in these studies, and one may argue that lipid metabolism could have been altered by metabolic changes due to the breast cancer disease [38].

In the present study, we observed a protective effect of the HDL-cholesterol/total-cholesterol ratio on overall mortality only among the TNBC patients. We have recently observed that low HDL-cholesterol is associated with more aggressive tumor characteristics [29]. A low level of HDL-cholesterol has been inversely associated with the activity of the pro-inflammatory cytokine interleukin (IL)-6 [39]. Moreover, IL-6 and IL-8 may promote tumor progression in TNBC cells [40]. Interestingly, it has been proposed that HDL-cholesterol possess anti-tumorigenic properties through regulation of angiogenesis, involving lowered expression of vascular endothelial growth factor (VEGF) [41]. Studies show that high expression of VEGF correlates with metastatic potential of TNBC [42], suggesting a potential biological link between low HDL-cholesterol and tumor progression among these patients. There are plausible biologic mechanisms supporting a role of triglycerides in tumor proliferation, growth and metastasis among patients with TNBC: Triglycerides play a role in energy storage, and they serve as a source for fatty acid oxidation (FAO), an important energy source for cell proliferation and migration. Overexpressed FAO is associated with more aggressive tumors [43], and studies show that metastatic TNBC cell lines maintain high levels of ATP through up-regulated fatty acid oxidation [44]. Triglyceride/HDL ratio may serve as a surrogate marker of insulin resistance [35], and insulin resistance further stimulates triglyceride production through lipolysis, and de novo fatty acid synthesis in the liver [45]. Both insulin resistance and triglycerides correlate with presence of white adipose tissue (WAT) inflammation in the breast, a proposed link between chronic subclinical inflammation, breast cancer aggressiveness and worse breast cancer prognosis [46]. This correlation remains independent of patients ' BMI [47], suggesting WAT inflammation and associated metabolic obesity, including high triglycerides, is a stronger driver of breast cancer progression than obesity alone.

We observed an inverse association between triglycerides and overall mortality among patients overexpressing HER2. This observation is in contrast to some previous 
observations on HER2+ tumors which suggest a reduced tumor proliferation from the inhibitory effect of polyunsaturated fatty acids on fatty acid synthase [48, 49]. While being inversely associated to serum levels of polyunsaturated fatty acids, triglyceride levels correspond to serum levels of the saturated fatty acid, palmitate [50], and studies show that endogeneous palmitate may be toxic to HER2+ cells [51]. Therefore, a deeper understanding of the biologic mechanisms underlying these results are needed.

Our study has several strengths. Although we can not fully exclude selection and diagnostic bias, the high attendance rate $(77 \%)$ in the population-based Tromsø study and completeness of identification of breast cancer cases due to mandatory registration of all new cases through the Cancer Registry of Norway (historically $98.8 \%$ complete) [52], indicate that the results are highly representative of the source population. Additionally, continuous registration of death (Norwegian Cause of Death Registry) and emigration (Statistics of Norway) limits loss to follow-up and missing endpoint data. Moreover, all medical charts were systematically reviewed with tumor and treatment characteristics abstracted. A majority of the tumors' histopathology was reanalyzed by tissue microarrays [36], enabling complete tumor characteristics and comparison between invasive breast cancer cases and molecular subtypes diagnosed at various time points. We observed agreement between molecular subtyping based on immunohistochemistry and tissue microarrays, supporting our tissue microarrays reanalyzing. Moreover, our long follow-up (mean 8.4 years) increases the chance of registering late recurrences.

A limitation of our study is the small number of patients in each molecular subclass, and the study may be underpowered to detect the hypothesized differences in all the distinct subclasses, which underlines the need for additional studies. Furthermore, as a result of limited number of events and in accordance with other studies $[53,54]$, we used breast cancer-free interval instead of breast cancer specific mortality as one of our endpoints. In order to classify all cases into molecular subclasses, a total of 123 women were excluded due to missing information on Ki67. However, characteristics of these women did not substantially differ from the study population. Furthermore, patients were diagnosed during a wide time period (1980-2014) with possible secular treatment effect, but reanalyzing histological classifications and adjusting for year of diagnosis and adjuvant systemic treatment according to national uniform guidelines by the Norwegian Breast Cancer Group [http:// www.nbcg.no], did not influence our main results. Comorbidity may be a potential confounder when studying breast cancer survival, and missing information on comorbid disease can be a concern. We used s-glucose, blood pressure, BMI, physical activity, alcohol and smoking habits as markers of comorbid disease. However, additional data on comorbidity may potentially add valuable information to our results [55]. Blood samples were not collected in a fasting state, which can affect lipid levels, but we adjusted for time since last meal in our analyses. Although we adjusted for BMI, we could not adjust for other variables that affect lipid levels such as diet, genetics or familial predisposition, as the data were not available.

\section{Conclusions}

Our study supports an association between pre-diagnostic triglycerides and the HDL-cholesterol/total-cholesterol ratio with survival for TNBC breast cancer patients. High triglycerides may be a negative prognostic marker, while pre-diagnostic high HDL-cholesterol/total-cholesterol ratio suggests improved prognosis. These findings are supported by plausible biological mechanisms linking triglycerides and the HDL-cholesterol/total-cholesterol ratio to breast cancer growth and progression. TNBC is associated with poor prognosis, and identifying and incorporating clinically available biomarkers is of great importance in order to improve the outcomes for this subgroup of breast cancer patients. Additional and larger studies including molecular subtyping, as well as more detailed information on comorbidity, are needed to define the clinical implications of these findings. Our findings may encourage closer follow-up of women at risk and future clinical trials to test effects of lipid-altering medications on breast cancer prognosis.

\section{Additional files}

\begin{abstract}
Additional file 1: Table S1. Multivariable adjusted Cox proportional hazard ratios (HRs) for overall mortality $\left(\mathrm{n}_{\text {cases }}=114\right)$ and breast cancerfree interval $\left(n_{\text {cases }}=107\right.$ ) by pre-diagnostic triglycerides and HDL/totalcholesterol ratio among triple negative breast cancer (TNBC) patients in the imputed data set (DOCX $16 \mathrm{~kb}$ )
\end{abstract}

Additional file 2: Figure S1. Age-adjusted overall survival by breast cancer molecular subtypes ${ }^{a}$. ' Luminal A - ER positive, PR positive, HER2 negative, and Ki-67 < 20\%; Luminal B - ER positive and/or PR positive, HER2 positive (or HER2 negative and Ki- $67 \geq 20 \%$ or PR negative); HER2 enriched - ER negative, PR negative, and HER2 positive; and TNBC - ER negative, PR negative and HER2 negative. Abbreviations: ER, estrogen receptor; HER2, human epidermal growth factor receptor-2; PR, progesterone receptor; TNBC, triple negative breast cancer (PDF $215 \mathrm{~kb}$ )

Additional file 3: Figure S2. Age-adjusted breast cancer-free interval by breast cancer molecular subtypes a a Luminal A - ER positive, PR positive, HER2 negative, and Ki-67 < 20\%; Luminal B - ER positive and/or PR positive, HER2 positive (or HER2 negative and Ki-67 $\geq 20 \%$ or PR negative); HER2-enriched - ER negative, PR negative, and HER2 positive; and TNBC ER negative, PR negative and HER2 negative. Abbreviations: ER, estrogen receptor; HER2, human epidermal growth factor receptor-2; PR, progesterone receptor; TNBC, triple negative breast cancer (PDF 215 kb)

Additional file 4: Table S2. Multivariable adjusted Cox proportional hazard ratios (HRs) for overall mortality and breast cancer-free interval by pre-diagnostic triglycerides among HER2+ patients (DOCX $16 \mathrm{~kb}$ )

Additional file 5: Table S3. Multivariable adjusted Cox proportional hazard ratios (HRs) for overall mortality and breast cancer-free interval by 
pre-diagnostic triglycerides/HDL-cholesterol ratio among triple negative breast cancer (TNBC) patients (DOCX $16 \mathrm{~kb}$ )

\section{Abbreviations}

BMI: Body mass index; CCBIO: Centre for cancer biomarkers; Cl: Confidence interval; CV: Coefficient of variation; EBBA: Energy balance and breast cancer aspect; ER: Estrogen receptor; FAO: Fatty acid oxidation; HDL: High density lipoprotein; HER2: Human epidermal growth factor receptor2; HR: Hazard ratio; IHC: Immunohistochemistry; IL: Interleukin; n: Number; PR: Progesterone receptor; SISH: Silver in situ hybridization; TMA: Tissue microarray; VEGF: Vascular endothelial growth factor; WAT: White adipose tissue

\section{Acknowledgements}

We thank the participants in the municipality of Troms $\varnothing$ Northern Norway, The Institute of Community Medicine (University of Tromsø), The Department of Oncology (University Hospital of Northern Norway), and The Department of Pathology (Haukeland University Hospital, Bergen, Norway). The study has used data from the Cancer Registry of Norway; the interpretation and reporting of these data are the sole responsibility of the authors, and no endorsement by the Cancer Registry of Norway is intended nor should be inferred.

\section{Funding}

This work was supported by The Norwegian ExtraFoundation for Health and Rehabilitation (2013/FOM 5628), Norwegian Cancer Society (2012/2/0054). The funding had no role in the design of the study and collection, analysis, and interpretation of data and in writing the manuscript.

\section{Availability of data and materials}

The data material consists of sensitive information on an individual level. Due to protection of privacy and restrictions from the Norwegian Data Protection Authority and the Regional Committee for Medical and Health Research Ethics the data are not publicly available.

\section{Authors` contributions}

$T L$, JBR and IT conceived of the study. AEE contributed to intellectual content and interpretation of data from the Troms $\varnothing$ study. HF and TL abstracted clinical and histopathological data from medical charts. $\mathrm{TL}$ performed the statistical analysis and drafted the manuscript. TW contributed to the statistical analysis and interpretation of the data. ESM performed histological examinations and helped draft the manuscript. HN, SA and LAA performed tissue microarray reanalysis. EW, AS, and TR contributed with clinical expertise. AM, LA, JBR, and IT contributed to interpretation of the data and revised the manuscript critically for important intellectual content. All authors read and approved the final manuscript.

\section{Ethics approval and consent to participate}

All clinical data in the present study are part of the "The Energy Balance and Breast Cancer Aspects throughout life (EBBA)-life" study, approved by the Norwegian Regional Committee for Medical and Health Research Ethics (2015/599). The Tromsø Study has been approved by the Regional Committee for Medical and Health Research Ethics and the Norwegian Data Protection Authority. Informed consent was implied by participation. The Norwegian Regional Committee for Medical and Health Research Ethics approved that the EBBA-life study was carried out without new consent from the participants.

\section{Competing interests}

The authors declare that they have no competing interests.

\section{Publisher's Note}

Springer Nature remains neutral with regard to jurisdictional claims in published maps and institutional affiliations.

\section{Author details}

'Department of Oncology, Oslo University Hospital, Ullevål, N-0424 Oslo, Norway. ${ }^{2}$ Department of Clinical Pathology, University Hospital of North Norway, N-9019 Tromsø, Norway. ${ }^{3}$ Centre for Cancer Biomarkers CCBIO, Department of Clinical Medicine, University of Bergen, N-5007 Bergen, Norway. ${ }^{4}$ Department of Community Medicine, Faculty of Health Services,
UIT The Arctic University of Norway, N-9019 Troms $\varnothing$, Norway. ${ }^{5}$ Department of Oncology, University Hospital of North Norway, N-9019 Tromsø, Norway. ${ }^{6}$ Fred Hutchinson Cancer Research Center, Public Health Sciences Division, Seattle, WA 98109, USA. ${ }^{7}$ Department of Oncology, Østfold Hospital Trust, N-1714 Grålum, Norway. ${ }^{8}$ Department of Pathology, Haukeland University Hospital, N-9019 Bergen, Norway. ${ }^{9}$ Institute of Clinical Medicine, Faculty of Health Services, UIT The Arctic University of Norway, N-9019 Tromsø, Norway.

Received: 27 June 2017 Accepted: 31 May 2018

Published online: 15 June 2018

\section{References}

1. Azrad M, Demark-Wahnefried W. The association between adiposity and breast cancer recurrence and survival: a review of the recent literature. Curr Nutr Rep. 2014;3(1):9-15.

2. Luo J, Virnig B, Hendryx M, Wen S, Chelebowski R, Chen C, Rohan T, Tinker L, Wactawski-Wende J, Lessin L, et al. Diabetes, diabetes treatment and breast cancer prognosis. Breast Cancer Res Treat. 2014;148(1):153-62.

3. Bahl M, Ennis M, Tannock IF, Hux JE, Pritchard Kl, Koo J, Goodwin PJ. Serum lipids and outcome of early-stage breast cancer: results of a prospective cohort study. Breast Cancer Res Treat. 2005;94(2):135-44.

4. Wulaningsih W, Vahdaninia M, Rowley M, Holmberg L, Garmo H, Malmstrom H, Lambe M, Hammar N, Walldius G, Jungner I, et al. Prediagnostic serum glucose and lipids in relation to survival in breast cancer patients: a competing risk analysis. BMC Cancer. 2015;15:913.

5. Prat A, Pineda E, Adamo B, Galvan P, Fernandez A, Gaba L, Diez M, Viladot M, Arance A, Munoz M. Clinical implications of the intrinsic molecular subtypes of breast cancer. Breast (Edinburgh, Scotland). 2015: 24(Suppl 2):S26-35.

6. Braunstein LZ, Taghian AG. Molecular phenotype, multigene assays, and the Locoregional Management of Breast Cancer. Semin Radiat Oncol. 2016;26(1):9-16.

7. Anders CK, Abramson V, Tan T, Dent R. The evolution of triple-negative breast Cancer: from biology to novel therapeutics. Am Soc Clin Oncol Educ book / ASCO Am Soc Clin Oncol Meet. 2016;35:34-42.

8. Polyak K. Heterogeneity in breast cancer. J Clin Invest. 2011;121(10):3786-8.

9. Martinez-Outschoorn UE, Peiris-Pages M, Pestell RG, Sotgia F, Lisanti MP. Cancer metabolism: a therapeutic perspective. Nat Rev Clin Oncol. 2017; 14(1):11-31.

10. Cibeira GH, Giacomazzi J, Aguiar E, Schneider S, Ettrich B, Cl DES, Camey S, Caleffi M, Weber B, Ashton-Prolla P, et al. Apolipoprotein E genetic polymorphism, serum lipoprotein levels and breast cancer risk: a casecontrol study. Mol Clin Oncol. 2014;2(6):1009-15.

11. His M, Dartois L, Fagherazzi G, Boutten A, Dupre T, Mesrine S, BoutronRuault MC, Clavel-Chapelon F, Dossus L. Associations between serum lipids and breast cancer incidence and survival in the $\mathrm{E} 3 \mathrm{~N}$ prospective cohort study. Cancer Causes Control. 2017;28(1):77-88.

12. Gershuni V, Li YR, Williams AD, So A, Steel L, Carrigan E, Tchou J. Breast cancer subtype distribution is different in normal weight, overweight, and obese women. Breast Cancer Res Treat. 2017;

13. Agresti R, Meneghini E, Baili P, Minicozzi P, Turco A, Cavallo I, Funaro F, Amash $\mathrm{H}$, Berrino F, Tagliabue E, et al. Association of adiposity, dysmetabolisms, and inflammation with aggressive breast cancer subtypes: a cross-sectional study. Breast Cancer Res Treat. 2016;157(1): 179-89.

14. Kwan ML, Kroenke CH, Sweeney C, Bernard PS, Weltzien EK, Castillo A, Factor RE, Maxfield KS, Stijleman IJ, Kushi LH, et al. Association of high obesity with PAM50 breast cancer intrinsic subtypes and gene expression. BMC Cancer. 2015;15:278.

15. Duggan C, Irwin ML, Xiao L, Henderson KD, Smith AW, Baumgartner RN, Baumgartner KB, Bernstein L, Ballard-Barbash R, McTiernan A. Associations of insulin resistance and adiponectin with mortality in women with breast cancer. J Clin Oncol. 2011;29(1):32-9.

16. Calip GS, Malone KE, Gralow JR, Stergachis A, Hubbard RA, Boudreau DM. Metabolic syndrome and outcomes following early-stage breast cancer. Breast Cancer Res Treat. 2014;148(2):363-77.

17. Cespedes Feliciano EM, Kwan ML, Kushi LH, Chen WY, Weltzien EK, Castillo AL, Sweeney C, Bernard PS, Caan BJ. Body mass index, PAM50 subtype, recurrence and survival among patients with nonmetastatic breast cancer Cancer. 2017;123(13):2535-42. 
18. Tait S, Pacheco JM, Gao F, Bumb C, Ellis MJ, Ma CX. Body mass index, diabetes, and triple-negative breast cancer prognosis. Breast Cancer Res Treat. 2014;146(1):189-97.

19. Chen HL, Ding A, Wang ML. Impact of central obesity on prognostic outcome of triple negative breast cancer in Chinese women. SpringerPlus. 2016;5:594.

20. Balaban S, Lee LS, Schreuder M, Hoy AJ. Obesity and cancer progression: is there a role of fatty acid metabolism? Biomed Res Int. 2015;2015:274585.

21. Saavedra-Garcia P, Nichols K, Mahmud Z, Fan LY, Lam EW. Unravelling the role of fatty acid metabolism in cancer through the FOXO3-FOXM1 axis. Mol Cell Endocrinol. 2017. S0303-7207(17):30016-3.

22. Li X, Tang H, Wang J, Xie X, Liu P, Kong Y, Ye F, Shuang Z, Xie Z, Xie X. The effect of preoperative serum triglycerides and high-density lipoproteincholesterol levels on the prognosis of breast cancer. Breast (Edinburgh, Scotland). 2017:32:1-6.

23. Warner M, Gustafsson JA. On estrogen, cholesterol metabolism, and breast cancer. N Engl J Med. 2014;370(6):572-3.

24. His M, Zelek L, Deschasaux M, Pouchieu C, Kesse-Guyot E, Hercberg S, Galan P, Latino-Martel P, Blacher J, Touvier M. Prospective associations between serum biomarkers of lipid metabolism and overall, breast and prostate cancer risk. Eur J Epidemiol. 2014;29(2):119-32.

25. Furberg AS, Veierod MB, Wilsgaard T, Bernstein L, Thune I. Serum highdensity lipoprotein cholesterol, metabolic profile, and breast cancer risk. J Natl Cancer Inst. 2004;96(15):1152-60.

26. Furberg AS, Jasienska G, Bjurstam N, Torjesen PA, Emaus A, Lipson SF, Ellison PT, Thune I. Metabolic and hormonal profiles: HDL cholesterol as a plausible biomarker of breast cancer risk. The Norwegian EBBA study. Cancer Epidemiol Biomark Prev. 2005;14(1):33-40.

27. Flote VG, Frydenberg H, Ursin G, Iversen A, Fagerland MW, Ellison PT, Wist EA, Egeland T, Wilsgaard T, McTiernan A, et al. High-density lipoproteincholesterol, daily estradiol and progesterone, and mammographic density phenotypes in premenopausal women. Cancer Prev Res (Phila). 2015;8(6): 535-44

28. Emaus A, Veierod MB, Tretli S, Finstad SE, Selmer R, Furberg AS, Bernstein L, Schlichting E, Thune I. Metabolic profile, physical activity, and mortality in breast cancer patients. Breast Cancer Res Treat. 2010;121(3):651-60.

29. Flote VG, Vettukattil $R$, Bathen TF, Egeland T, McTiernan A, Frydenberg $H$, Husoy A, Finstad SE, Lomo J, Garred O, et al. Lipoprotein subfractions by nuclear magnetic resonance are associated with tumor characteristics in breast cancer. Lipids Health Dis. 2016;15:56.

30. Fan Y, Ding X, Wang J, Ma F, Yuan P, Li Q, Zhang P, Xu B. Decreased serum HDL at initial diagnosis correlates with worse outcomes for triple-negative breast cancer but not non-TNBCs. Int J Biol Markers. 2015;30(2):e200-7.

31. Eggen AE, Mathiesen EB, Wilsgaard T, Jacobsen BK, Njolstad I. The sixth survey of the Tromso study (Tromso 6) in 2007-08: collaborative research in the interface between clinical medicine and epidemiology: study objectives, design, data collection procedures, and attendance in a multipurpose population-based health survey. Scand J Public Health. 2013;41(1):65-80.

32. Braekkan SK, Hald EM, Mathiesen EB, Njolstad I, Wilsgaard T, Rosendaal FR, Hansen JB. Competing risk of atherosclerotic risk factors for arterial and venous thrombosis in a general population: the Tromso study. Arterioscler Thromb Vasc Biol. 2012;32(2):487-91.

33. Aziz S, Wik E, Knutsvik G, Klingen TA, Chen Y, Davidsen B, Aas H, Aas T, Akslen LA. Evaluation of tumor cell proliferation by Ki-67 expression and mitotic count in lymph node metastases from breast Cancer. PLoS One. 2016;11(3):e0150979

34. Goldhirsch A, Winer EP, Coates AS, Gelber RD, Piccart-Gebhart M, Thurlimann B, Senn HJ. Personalizing the treatment of women with early breast cancer: highlights of the St Gallen international expert consensus on the primary therapy of early breast Cancer 2013. Ann Oncol. 2013;24(9):2206-23

35. McLaughlin T, Reaven G, Abbasi F, Lamendola C, Saad M, Waters D, Simon J, Krauss RM. Is there a simple way to identify insulin-resistant individuals at increased risk of cardiovascular disease? Am J Cardiol. 2005;96(3):399-404.

36. Knutsvik G, Stefansson IM, Aziz S, Arnes J, Eide J, Collett K, Akslen LA. Evaluation of Ki67 expression across distinct categories of breast cancer specimens: a population-based study of matched surgical specimens, core needle biopsies and tissue microarrays. PLoS One. 2014;9(11):e112121.

37. Dai D, Chen B, Wang B, Tang H, Li X, Zhao Z, Li X, Xie X, Wei W. Pretreatment TG/HDL-C ratio is superior to triacylglycerol level as an independent prognostic factor for the survival of triple negative breast Cancer patients. J Cancer. 2016;7(12):1747-54

38. Kinlaw WB, Baures PW, Lupien LE, Davis WL, Kuemmerle NB. Fatty acids and breast Cancer: make them on site or have them delivered. J Cell Physiol. 2016;231(10):2128-41.

39. Holven KB, Retterstol K, Ueland T, Ulven SM, Nenseter MS, Sandvik M, Narverud I, Berge KE, Ose L, Aukrust P, et al. Subjects with low plasma HDL cholesterol levels are characterized by an inflammatory and oxidative phenotype. PLoS One. 2013;8(11):e78241.

40. Hartman ZC, Poage GM, den Hollander P, Tsimelzon A, Hill J, Panupinthu N, Zhang Y, Mazumdar A, Hilsenbeck SG, Mills GB, et al. Growth of triple-negative breast cancer cells relies upon coordinate autocrine expression of the proinflammatory cytokines IL-6 and IL-8. Cancer Res. 2013;73(11):3470-80.

41. Tan JT, Ng MK, Bursill CA. The role of high-density lipoproteins in the regulation of angiogenesis. Cardiovasc Res. 2015;106(2):184-93.

42. Roberti MP, Arriaga JM, Bianchini M, Quinta HR, Bravo Al, Levy EM, Mordoh J, Barrio MM. Protein expression changes during human triple negative breast cancer cell line progression to lymph node metastasis in a xenografted model in nude mice. Cancer Biol Ther. 2012;13(11):1123-40.

43. Wang YY, Attane C, Milhas D, Dirat B, Dauvillier S, Guerard A, Gilhodes J, Lazar I, Alet N, Laurent $\mathrm{V}$, et al. Mammary adipocytes stimulate breast cancer invasion through metabolic remodeling of tumor. Cell. 2017;2(4):e87489.

44. Park JH, Vithayathil S, Kumar S, Sung PL, Dobrolecki LE, Putluri V, Bhat VB, Bhowmik SK, Gupta V, Arora K, et al. Fatty acid oxidation-driven Src links mitochondrial energy reprogramming and oncogenic properties in triplenegative breast Cancer. Cell Rep. 2016;14(9):2154-65.

45. Miccoli. Insulin resistance and lipid disorder. In: Future Lipidology; 2008.

46. lyengar NM, Zhou XK, Gucalp A, Morris PG, Howe LR, Giri DD, Morrow M, Wang H, Pollak M, Jones LW, et al. Systemic correlates of white adipose tissue inflammation in early-stage breast Cancer. Clin Cancer Res. 2016;22(9):2283-9.

47. Iyengar NM, Brown KA, Zhou XK, Gucalp A, Subbaramaiah K, Giri DD, Zahid H, Bhardwaj P, Wendel NK, Falcone DJ, et al. Metabolic obesity, adipose inflammation and elevated breast aromatase in women with normal body mass index. Cancer prevention research (Philadelphia, Pa). 2017;10(4):235-43.

48. Mashima $T$, Seimiya $H$, Tsuruo $T$. De novo fatty-acid synthesis and related pathways as molecular targets for cancer therapy. $\mathrm{Br} J$ Cancer. 2009;100(9):1369-72.

49. Teran-Garcia M, Adamson AW, Yu G, Rufo C, Suchankova G, Dreesen TD, Tekle M, Clarke SD, Gettys TW. Polyunsaturated fatty acid suppression of fatty acid synthase (FASN): evidence for dietary modulation of NF-Y binding to the Fasn promoter by SREBP-1C. Biochem J. 2007:402(3):591-600.

50. Lopez-Alvarenga JC, Ebbesson SO, Ebbesson LO, Tejero ME, Voruganti VS, Comuzzie AG. Polyunsaturated fatty acids effect on serum triglycerides concentration in the presence of metabolic syndrome components. The Alaska-Siberia project. Metab Clin Exp. 2010;59(1):86-92.

51. Baumann J, Wong J, Sun Y, Conklin DS. Palmitate-induced ER stress increases trastuzumab sensitivity in HER2/neu-positive breast cancer cells. BMC Cancer. 2016;16:551.

52. Larsen IK, Smastuen M, Johannesen TB, Langmark F, Parkin DM, Bray F, Moller B. Data quality at the Cancer registry of Norway: an overview of comparability, completeness, validity and timeliness. Eur JCancer (Oxford, England: 1990). 2009;45(7):1218-31.

53. Frydenberg $H$, Thune I, Lofterod $T$, Mortensen ES, Eggen AE, Risberg T, Wist EA, Flote VG, Furberg AS, Wilsgaard T, et al. Pre-diagnostic high-sensitive Creactive protein and breast cancer risk, recurrence, and survival. Breast Cancer Res Treat. 2016;155(2):345-54.

54. Hudis CA, Barlow WE, Costantino JP, Gray RJ, Pritchard KI, Chapman JA Sparano JA, Hunsberger S, Enos RA, Gelber RD, et al. Proposal for standardized definitions for efficacy end points in adjuvant breast cancer trials: the STEEP system. J Clin Oncol. 2007;25(15):2127-32.

55. Ewertz M, Land LH, Dalton SO, Cronin-Fenton D, Jensen MB. Influence of specific comorbidities on survival after early-stage breast cancer. Acta Oncol (Stockholm, Sweden). 2018;57(1):129-34. 